\title{
El desempleo y subempleo juvenil asociado a la baja calificación técnica de los jóvenes egresados de secundaria. Estudio de caso del Distrito Central período 1990-2006
}

Merlin Ivania Padilla Contreras ${ }^{1}$

\section{RESUMEN}

Este estudio tiene como objetivo general, evaluar las características del desempleo y subempleo juvenil en el Distrito Central y su relación con la situación actual de la educación técnica como medio para la inserción laboral de los jóvenes egresados de secundaria. Los objetivos específicos incluyen conocer la situación actual de la educación técnica y tecnológica de los jóvenes egresados de secundaria, la evaluación de la infraestructura físico/pedagógica de los centros de educación técnica y el nivel académico de los docentes, así como la evaluación de la magnitud y tendencias del desempleo juvenil en el Distrito Central.

El objeto de estudio, el desempleo y subempleo juvenil, está relacionado con muchos factores que tienen que ver con causas estructurales propias del modelo de acumulación capitalista, tal es el caso de las enormes masas de desempleados y desalentados que hacen contrapeso en las condiciones de empleabilidad de los que están empleados. Por otro lado la calificación técnica influye definitivamente en la inserción y la permanencia en un empleo de calidad. El estudio logra establecer esta relación mediante el análisis de la formación profesional de nivel medio que reciben los jóvenes en un periodo de 3 quinquenios en donde las tendencias apuntan a una tercerización de la economía y de la formación misma, pero en condiciones de precariedad e inestabilidad laboral. Por otro lado esta calificación técnica tiene sus causas en el mismo sistema educativo y particularmente en el nivel de educación media que históricamente ha estado desarticulado del resto de los niveles educativos por ser considerado una etapa de transición entre el estudio y el trabajo. El estudio permite concluir que la mitad de los docentes de educación media no ostentan títulos formales para ejercer, lo cual impacta directamente sobre la calidad de enseñanza. Los colegios técnicos utilizados en la muestra de este estudio presentan características específicas de formación profesional; pero no cuentan con políticas institucionales de inserción laboral que facilite a los alumnos el acceso y permanencia en un centro de trabajo. Todos estos factores repercuten en la calidad de los puestos de trabajo que son ocupados por los jóvenes y dificulta

\footnotetext{
${ }^{1}$ Universidad Nacional Autónoma de Honduras. Maestría en Economía y Desarrollo. ivaniapadilla@unah.edu.hn
} 
su acumulación de experiencia laboral y otras destrezas que aseguren un mejor puesto de trabajo en el futuro.

Palabras clave: desempleo juvenil, inserción laboral de jóvenes, calificación técnica, precarización laboral.

\section{ABSTRACT}

The general goal of the following research study is to evaluate the characteristics of youth unemployment and under employment in the Central District, and its relationship with current technical education situation as a medium to insert the high school graduating youth into the labor market. Specific goals include assessing the current situation of technological studies from high school graduates, to evaluate the physical/pedagogical infrastructure of centers for technical studies, and the teacher's academic level, as well as to evaluate the magnitude and trends of youth unemployment in the Central District.

The research subject, youth unemployment and sub-employment is related to many factors linked with structural causes of the capitalist wealth accumulation model, as is the case with the huge mass of unemployed bringing counter weight to the employment conditions of those that have jobs. On the other hand, technical qualification definitely influences quality job insertion and permanence. The study establishes this relationship via the analysis of the youth's professional education at high school level during three five year periods in which trends aims at the outsourcing of economy and education itself, but in conditions of precarious and unstable jobs. This technical qualification has its roots in the educational system itself, particularly on the quality of high school education that has been historically non articulated with the rest of educational levels, being considered a transition phase between studies and labor market. The study concludes that half the teachers of high school education do not have formal qualifications to teach, which directly impacts teaching quality. Technological schools observed as a sample for the study do show characteristics of professional formation, but do not count with institutional policies for job insertion that would ease youth access to permanent jobs. All these factors impact on the quality of the jobs occupied by youth and makes difficult the accumulation of work experience and other abilities that would ensure a better job in their future.

Key words: youth unemployment, youth insertion in labor force, technological qualifications, jobs outsourcing. 


\section{INTRODUCCIÓN}

El desempleo y subempleo juvenil asociado a la baja calificación técnica de los jóvenes egresados de secundaria, surge como una iniciativa de tesis de grado para optar al Titulo de Máster en Economía y Desarrollo del POSCAE en noviembre del 2007. Las tendencias del desempleo y subempleo juvenil en el contexto hondureño, revelan una problemática aguda y compleja fundamentalmente de carácter estructural, que obliga a la investigación y sistematización de sus causas.

Una vez que se desvelan las causas del fenómeno del desempleo juvenil, se pueden generar propuestas para superarlo. En consecuencia, con los resultados de este estudio se puede definir una propuesta de empleabilidad juvenil más eficaz.

De igual manera, esta investigación puede servir de referencia para orientar la actual política educativa hacia una inserción laboral más eficiente, incluyente y diversificada, congruente con las necesidades económicas y sociales del país.

Finalmente la importancia que tiene el estudio de un fenómeno tan dramático como el desempleo y subempleo juvenil, permite la inclusión de una metodología de investigación exhaustiva y sistemática, por lo que el estudio sirve también como fuente orientadora de los procesos de investigación y análisis, en el desarrollo académico hondureño.

\section{METODOLOGÍA DE LA INVESTIGACIÓN}

Este estudio utiliza como fuentes secundarias todas la Encuestas Permanentes de Hogares elaboradas por el Instituto Nacional de Estadística desde 1990, finalizando con la XXXIV de mayo del 2007, para obtener los valores de cada variable de forma histórica y construir las relaciones necesarias.

Con estas encuestas se obtendrán valores importantes de comparación para la población entre los 15-24 años del Distrito Central, no obstante que en algunas encuestas se incluyen los jóvenes entre los 25 y 29 años; en estos casos se hará la respectivamención.

Asimismo, se utilizarán las estadísticas recopiladas por la Secretaría de Educación Pública para construir las series históricas del total de institutos en el Distrito Central, el número de docentes empleados, los niveles de matrícula y obtener datos sobre los presupuestos destinados a la educación secundaria. Para el cálculo de 
cifras se ha utilizado tanto el programa SPSS 15.0 versión en español, como el programa Excel 2007.

Como fuentes primarias, se desarrolló una encuesta para conocer la situación de los institutos técnicos en el Distrito Central, y para seleccionar dicha muestra se tomó en cuenta el criterio de nivel de matricula. La encuesta aplicada contiene varios elementos provenientes del estudio realizado por Gómez, V. (1998) hecho con los mismos propósitos en Colombia sobre la educación industrial.

Los siete colegios seleccionados son de reconocida trayectoria en la educación técnica del sector público y se incluyó para efectos de comparación un colegio técnico del sector privado. La metodología efectiva consistió en hacer la visita al Director (a) del instituto, y realizar las entrevistas que incluían un recorrido por las instalaciones y que se documenta con fotografías de las mismas.

\section{ELDESEMPLEOY SUBEMPLEO JUVENIL}

En el Distrito Central, la población juvenil crece al ritmo de la población total, y como parte de esa población enfrenta el problema de desempleo. El grupo de jóvenes entre los 15 y 24 años representan la quinta parte de esa población, lo que totaliza 216 mil jóvenes capitalinos y aproximadamente 29 por cada 100 personas en edad de trabajar se encuentran dentro del rango de edad antes mencionado (INE, 2007).

La población juvenil que se encuentra efectivamente trabajando cubre en promedio el 56 por ciento de la población juvenil total, y representa el 42 por ciento de la PEA total del Distrito Central, durante el periodo 1990-2006. Según las cifras anteriores, más de 129 mil jóvenes con edad para trabajar, estaban fuera del mercado laboral en el 2006.

No es casualidad que la tasa de desempleo juvenil sea más pronunciada que la adulta y nacional. Por lo general son los jóvenes los que obtienen la peor parte en el desempleo así como en los niveles de ocupación, debido a múltiples razones: falta de experiencia, falta de antigüedad, recesión económica, etc., todo lo cual afecta la disponibilidad de puestos de trabajo duraderos y de calidad para este segmento de la población. 
Gráfico No. 1

Distrito Central

Tasa de Desempleo Abierto Jóvenes 15-24 años. 1990-2006

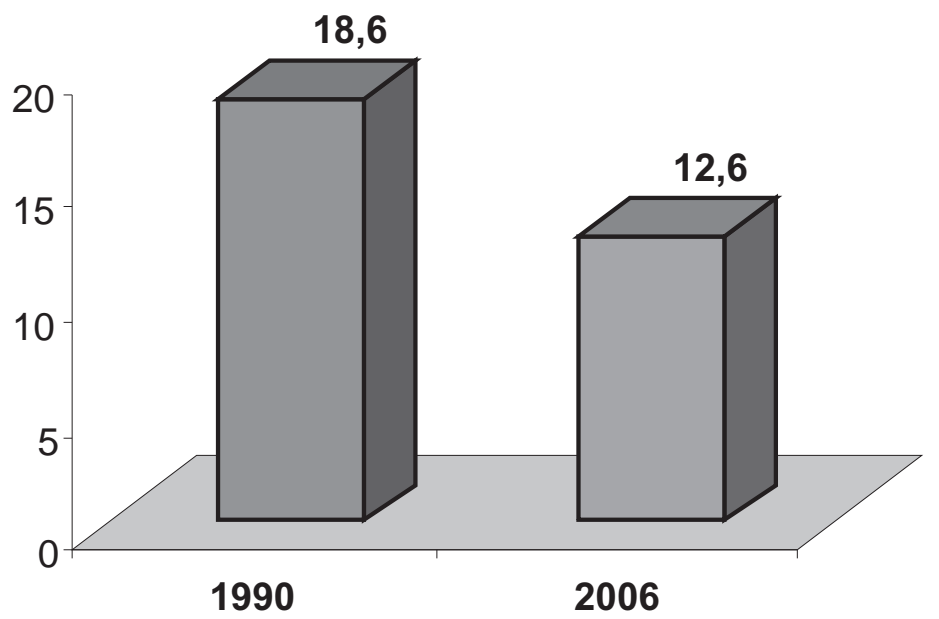

Fuente: ONU (2007) Objetivos de Desarrollo del Milenio. Informe de país 200, p. 141.

A pesar de que la tasa de desempleo abierto en jóvenes de 15 a 24 años ha disminuido entre 1990 y el 2006, aún es una cifra importante, tomando en cuenta la presencia de subempleo visible e invisible en el país. Son más de 30 mil jóvenes desempleados que significa un desperdicio de talento humano y mayores niveles de dependencia y pobreza entre la población capitalina, debido a la inexistencia de programas asistenciales o de seguro de desempleo en el país. En relación a las tasas de desempleo por género, las mujeres presentan tasas mayores de desempleo abierto con relación a los hombres, al igual que sucede con la población adulta, lo que significa que sufren más inestabilidad en el empleo que obtienen. Por si fuera poco la diferencia según dominio ahonda las brechas entre hombres y mujeres. Por otro lado, el nivel de instrucción está inversamente relacionado con el nivel de desempleo; lo anterior puede significar disminuir el riesgo de estar desempleado para los jóvenes que alcanzan niveles elevados de instrucción formal.

La comparación de los niveles educativos de la década de los 90 con relación al 2006, demuestra que el nivel educativo de los jóvenes pasa de la educación primaria a la educación secundaria, es decir, de 7 a 9 años de escolaridad en promedio. No obstante, los mejores niveles educativos no garantizan por sí mismos la inserción laboral en los jóvenes. Las tasas de desempleo abierto según el nivel educativo lo confirman. 
Gráfico No. 2

Honduras Tasa de desempleo abierto según nivel educativo Jóvenes 15 a 24 años

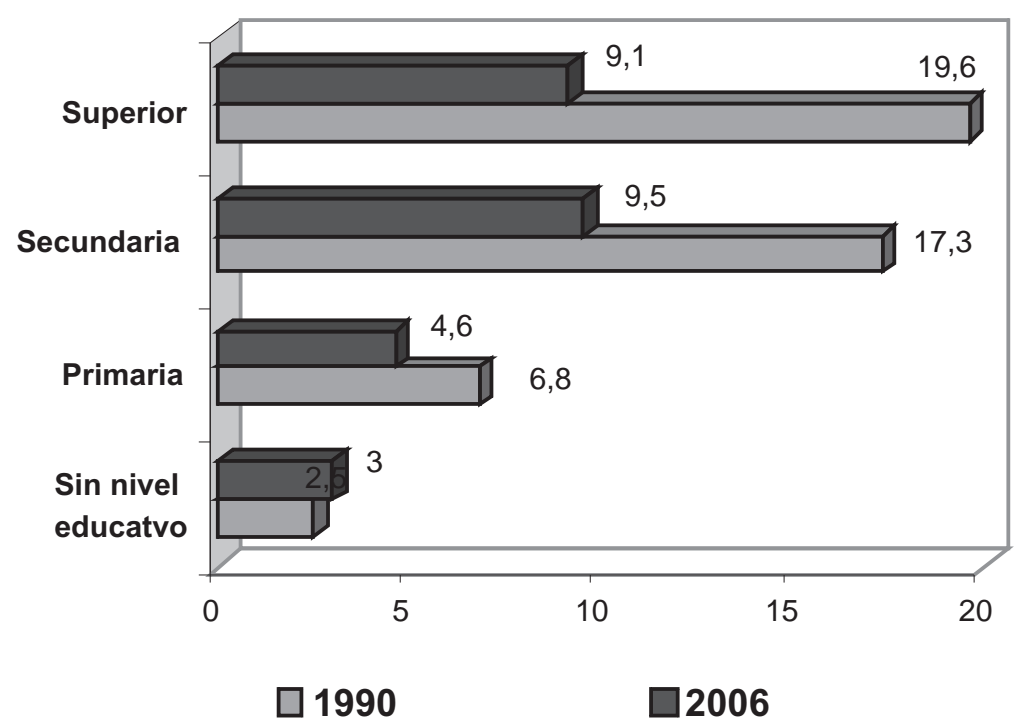

Fuente: Elaboración propia con datos de ONU (2007, p.141). Objetivos de Desarrollo del Milenio.

A pesar que de 1990 al 2006 se han reducido las tasas de desempleo abierto, excepto para los que no tienen ningún nivel educativo, estas cifras constituyen segmentos importantes de la población juvenil. La tasa de desempleo mayor la presenta la categoría de nivel medio aunque durante el periodo analizado se haya reducido en 8 puntos porcentuales.

En el caso del desempleo del profesional universitario, la tendencia de bajo desarrollo del capitalismo en el país es responsable de las elevadas cifras de desempleados entre estos profesionales, al no lograr mantener una demanda sostenida de personal altamente calificado. Otro factor causal puede ser la poca pertinencia de carreras profesionales con relación a la economía laboral del país.

\section{El subempleo juvenil en el Distrito Central}

El fenómeno del subempleo afecta mayormente la población joven empleada. La TSV entre los jóvenes de 15 a 24 años en el 2006 fue de 6,1 en tanto la TSI llegó a 28,6 por ciento en ese mismo año, ambas mayores a las tasas a nivel nacional. La trascendencia del subempleo en los jóvenes radica en las desventajas que arrastra 
consigo, que pueden ser la empleabilidad en condiciones de informalidad, la subutilización de las capacidades y habilidades, la ausencia de contratos laborales, salarios bajos, negación de la cobertura de seguridad y salud laboral, entre otros.

Con respecto a las condiciones laborales, según los datos de la encuesta, al menos el 75 por ciento de los jóvenes trabajadores no tienen ningún tipo de beneficio social, como fondo de jubilaciones, seguro social, afiliación sindical, entre otros. En relación con el ingreso monetario promedio, éste resultó de Lps. 3, 369. 78 y el 50 por ciento de los jóvenes ganaba menos de tres mil lempiras mensuales.

\section{Cuadro No.1 \\ Distrito Central \\ Porcentaje de jóvenes 15-24 años según cotización y derechos laborales 2006}

\begin{tabular}{|c|c|c|c|}
\hline $\begin{array}{l}\text { Tipo de } \\
\text { Cotización }\end{array}$ & \% Jóvenes & Derechos Laborales & \% Jóvenes \\
\hline RAP & 4,6 & Prestaciones & 29 \\
\hline INJUPEM & 0,6 & Vacaciones & 32 \\
\hline INPREMA & 0,4 & Horas Extras & 12,9 \\
\hline IPM & 0,1 & Seguro Accidente & 6 \\
\hline IHSS & 12,3 & Aguinaldo & 37,3 \\
\hline $\begin{array}{l}\text { Seguro Médico } \\
\text { Privado }\end{array}$ & 1,2 & Catorceavo & 35,9 \\
\hline Sindicato & 0,4 & Bonificación & 5,6 \\
\hline Gremio & 0,2 & Seguro de Vida & 6,3 \\
\hline $\begin{array}{l}\text { Ninguna de las } \\
\text { anteriores }\end{array}$ & 76,4 & $\begin{array}{l}\text { Ninguna de las } \\
\text { anteriores }\end{array}$ & 75,3 \\
\hline
\end{tabular}

Fuente: Elaboración propia con datos de la XXXIV EPHPM 2007, INE.

Otro dato que revela la estabilidad y calidad del empleo es el tipo de establecimiento económico que lo provee. Para el año 2006, un 43 por ciento de los jóvenes entre los 15 y 24 años del Distrito Central trabajaban en establecimientos de menos de 10 trabajadores permanentes y el restante en empresas de más de 10 trabajadores (INE, 2007).

\section{LACALIFICACIÓN TÉCNICADE LOS EGRESADOS DE SECUNDARIA}

El trabajador con educación tiene mayores probabilidades de tener un buen rendimiento laboral y de aprender procedimientos y procesos nuevos con mayor 
facilidad que los que no ostentan esa educación. En el modelo económico de acumulación capitalista, el mayor beneficiario de esta ventaja laboral es el propietario del medio de producción, pues el incremento de la productividad genera mayor ganancia.

El gráfico siguiente muestra la distribución de las ocupaciones que desempeñan los jóvenes del Distrito Central. Los sectores de trabajo en la industria textil, servicios técnicos, de albañilería, mecánica, los comerciantes y vendedores y la ocupación de servicios, agrupan a más del 60 por ciento de los trabajadores de 15 a 24 años.

\section{Gráfico No. 3}

Distrito Central

Distribución de los Jóvenes 15-24 años según ocupación 2006

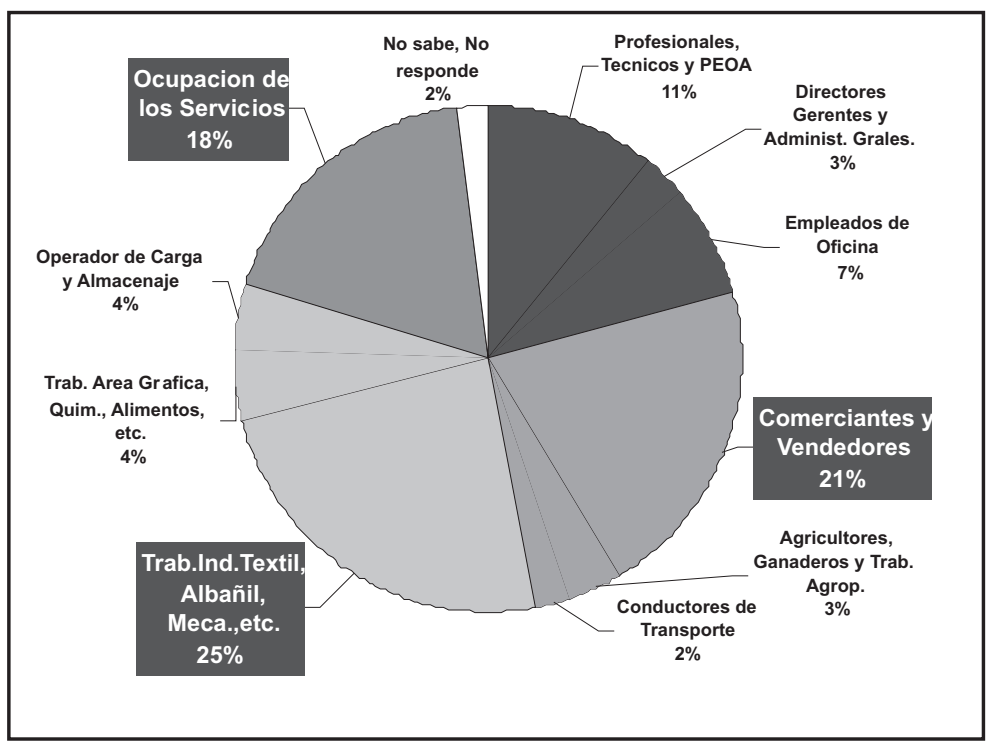

Fuente: Elaboración propia con datos de XXXIV EPHPM. INE.

Aunado a esto, más del 60 por ciento de los jóvenes con educación media empleados en el Distrito Central ostentan empleos de nivel medio y técnico, según la encuesta antes referida. Entonces tomando en consideración los hallazgos de desempleo y subempleo juvenil que son mayoritariamente en jóvenes con educación secundaria, así como la tendencia de las ocupaciones de los jóvenes trabajadores, se hace necesario el análisis de la calificación profesional que el nivel educativo secundario, específicamente la educación media, está dejando entre los estudiantes. 


\section{La educación media en Honduras}

La educación media se agrupa de la siguiente manera:

a) Modalidad Académica: comprende el Bachillerato en Ciencias y Letras de 2 años de duración y la cual ofrece formación científica y humanista, orientada a continuar estudios en el nivel superior.

b) Modalidad Profesional: con una duración de 2 ó 3 años, la cual permite al estudiante obtener una profesión para accesar al mundo laboral. Esta modalidad contiene una serie de subgrupos, descritos a continuación: b.1 Educación Agropecuaria, b.2 Educación industrial, b.3 Servicios Administrativos, b.4 Educación ambiental, turismo y salud

La finalidad de la educación media es preparar al educando para el nivel universitario y para el mercado laboral. Es decir, tiene una finalidad bivalente. Entre los objetivos, destacan la formación de competencias y habilidades para el trabajo y la investigación y aprendizaje continuo.

Según Salgado y Soleno (2002), la educación media se enfrenta con singulares retos para cumplir con su finalidad, y desde la perspectiva de la inserción laboral debe resolver asuntos como el avance vertiginoso y constante de las tecnologías, las nuevas formas de organización del trabajo y los escenarios económicos que exigen movilidad y polivalencia laboral (p.86-89).

La inclinación a considerar la modalidad profesional como una alternativa para el estudiantado pobre, se ha acentuado de tal manera que los padres ven en esta modalidad una alternativa de sustento.

\section{La cobertura de la educación media}

La tasa de cobertura neta de la educación media asciende a 25.7 por ciento, siendo el Distrito Central el que presenta la mayor cobertura con relación al resto de las regiones. Esto tiene su explicación por la mayor concentración de centros educativos y modalidades. 


\section{Cuadro No. 2}

Honduras

Población de 16 a 18 años que asiste a un Centro de Enseñanza Diversificada.

Según dominio

\begin{tabular}{|c|c|c|c|}
\hline Categorías & Población & Asistencia & $\begin{array}{c}\text { Tasa de } \\
\text { Cobertura }\end{array}$ \\
\hline Total & 597,211 & 153,514 & 25.7 \\
\hline Dominio & & & \\
\hline Urbano & 271,488 & 104,084 & 38.3 \\
\hline Distrito Central & 70,917 & 29,332 & 41.3 \\
\hline San Pedro Sula & 40,483 & 16,395 & 40.5 \\
\hline Resto Urbano & 160,088 & 58,368 & 36.5 \\
\hline Rural & 325,723 & 49,430 & 15.2 \\
\hline
\end{tabular}

Fuente: Elaboración propia en base a EPHPM XXXVI Mayo 2008, INE.

Según la Secretaría de Educación, en 2006 Tegucigalpa concentró 227 institutos de educación media que atienden la modalidad presencial, de los 267 que se contabilizan a nivel de todo el departamento de Francisco Morazán, es decir que concentra el 85 por ciento de la oferta educativa de media en modalidad presencial (SE, 2006).

\section{La calidad de la educación media}

En el año 2003 se realizó un estudio por la Secretaría de Educación donde se evaluó las condiciones de infraestructura básica analizando variables como el material de construcción del instituto, los servicios básicos y de comunicación así como el equipamiento mínimo, donde se constató que al menos 5 de cada 10 institutos no tienen un laboratorio de computación.

Entre las principales carencias identificadas está el área de comunicación, ya que 4 de cada 10 institutos carece de un medio de comunicación telefónica; igual cifra no posee laboratorio para sus clases de ciencias, y 6 de cada 10 institutos no cuentan con talleres para impartir sus clases. Según el estudio existen 21 computadoras por instituto, además que no cuentan con datos que aseguren si se encuentran en buen estado. No contar con una biblioteca es básicamente una debilidad educativa y al menos la mitad de los centros educativos del nivel medio en Honduras carecen de este importante recurso de aprendizaje. 
Asimismo, el que 23 de 100 pupitres no estén en buenas condiciones, priva a cierto porcentaje de alumnos a recibir sus clases en condiciones adecuadas para su correcto proceso de aprendizaje. Por otro lado los indicadores de eficiencia como la tasa de repitencia, deserción y reprobación, se han utilizado para construir un índice de eficiencia que en el Departamento de Francisco Morazán para el 2003, resultó ser de 89,6 lo cual es mayor en 4 puntos porcentuales del Índice de Eficiencia nacional (SE, 2000).

En relación con los docentes de educación media, la distribución según las zonas geográficas no es equitativa. Los departamentos donde se concentran más del 55 por ciento de los docentes son Francisco Morazán (31\%), Cortés (17,6\%) y Atlántida (7,6\%). Los departamentos con menos representación de docentes son Gracias a Dios e Islas de la Bahía. Esta desigualdad coincide con la distribución geográfica de los institutos y de igual manera con las tasas de cobertura. El 50 por ciento de los docentes ostentan el título de profesor de educación media.

Gráfico No. 4

Formación Académica de los docentes de nivel medio

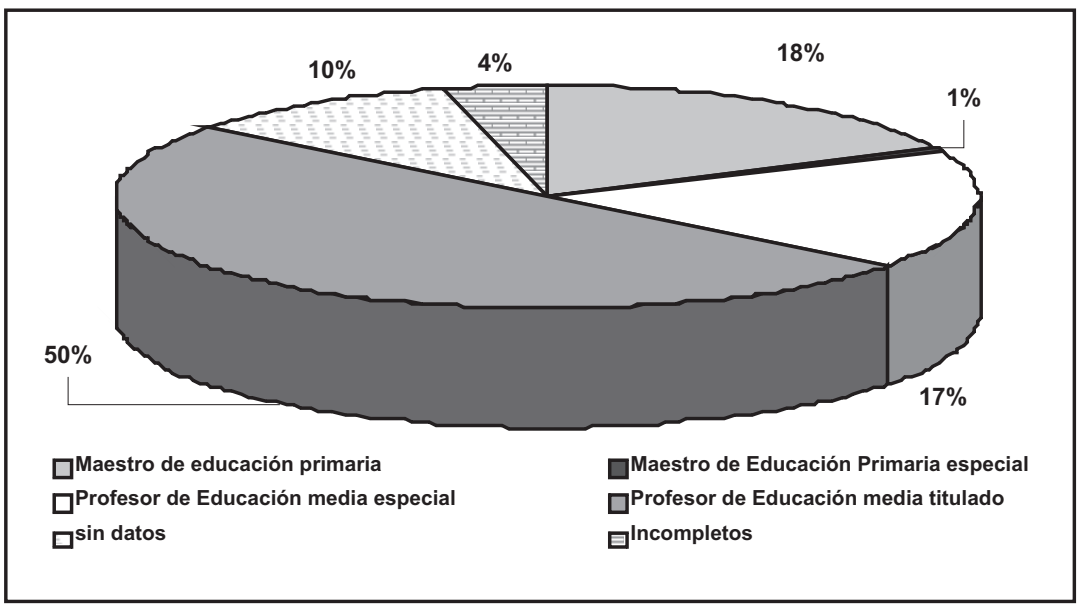

Fuente: Elaboración propia con datos de Hernández (2003).

Con relación a la evaluación docente, la Secretaría de Educación no cuenta con una política para este fin. Lo que se ha estilado en el quehacer docente es la supervisión de la planificación, trabajo en el aula y evaluación. El trabajo en el aula especialmente es poco supervisado y esta deficiencia es mayor en los colegios oficiales, semioficiales y pequeños institutos privados. (SE, 1996). Como parte importante de la calidad de la enseñanza secundaria, la cuestión de contar con un 
plan de estudios adecuado y pertinente constituye un elemento clave al que hay que prestar una atención especial en el nivel de la educación secundaria. Según estudios comparativos entre países, en Honduras se han identificado cuatro sectores estratégicos: el turismo, la agroindustria, la maquila y el forestal (SE, 2006, p.52). No obstante, la adecuación de los currículos de educación media ha respondido más a intereses políticos de algunos sectores y no a la realidad de las actividades económicas de las regiones. Actualmente hay más de 80 modalidades en este nivel. A nivel nacional, la comparación entre la modalidad profesional y la modalidad humanística refleja una brecha importante y permite confirmar la preferencia de los estudiantes por la modalidad profesional.

\section{Gráfico No. 5}

Matrícula de Educación Media según modalidad 1990-2006

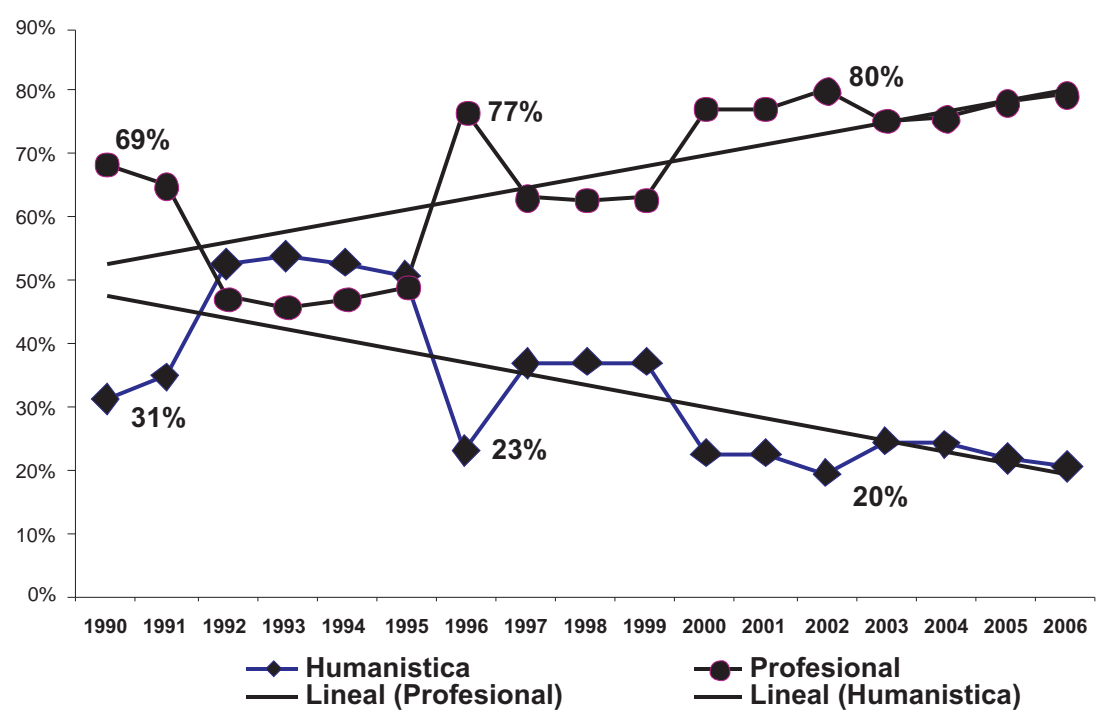

Fuente: Elaboración propia con datos de Honduras en Cifras 1990-2006 BCH.

En Francisco Morazán, las actividades donde están mayormente representados los empleados son en el comercio, la industria manufacturera y los servicios comunales y personales, las cuales concentran más del 50 por ciento de la fuerza de trabajo empleada. Ya se han visto las cifras de los jóvenes de 15 a 24 años empleados en su mayoría en el sector comercial y servicios. En cuanto a los egresados de las carreras de educación media en su orden, Perito Mercantil y Contador Público un 49.5 por ciento, Bachiller en Ciencias y Letras 16.2 por ciento y Bachillerato en Computación 9.3 por ciento, totalizando estas tres modalidades un 75 por ciento de los egresados (SE, 2006, p.61). Lo crucial en este punto es que, al ver las tendencias del país y del 
Distrito Central en particular, la educación secundaria es la última opción a la educación formal que la mayoría de la población tiene; preocupa en gran manera que la misma no tenga la calidad y pertinencia adecuadas para poder constituirse en la herramienta capaz de asegurarle un espacio en el mercado de trabajo. El exceso de egresados de estas modalidades permite que los empleadores mantengan salarios bajos y que exista una alta movilidad entre los mismos. Al no poseer estabilidad laboral tampoco acumulan beneficios ni mejoran sus salarios nominales.

\section{Políticas educativas en educación media}

En materia de políticas educativas, primeramente no se obedece a una articulación continua de estrategias, reflejada por la ruptura de los planes al finalizar el gobierno propulsor y entrar el nuevo. Los resultados de cada propuesta se ven a muy corto plazo aunque los impactos transciendan los años del gobierno en turno. En cuanto a la educación media se diagnosticó una problemática que luego no logra operativizar soluciones y hasta el 2006 se articula finalmente la política educativa con la laboral. A través de los estudios sobre la formación profesional y la adecuación curricular de la Educación Media, se visualiza una alternativa de reconversión de este nivel educativo tan importante para la vida económica y social del país.

\section{LA EDUCACIÓN TÉCNICA: ESTUDIO DE CASO DE COLEGIOS TÉCNICOS DELACAPITAL}

Los centros de educación técnica ubicados en la capital que fueron incluidos en la muestra son:

Cuadro No. 3

Información General de los institutos técnicos

\begin{tabular}{|l|r|}
\multicolumn{1}{c|}{ Nombre del Instituto } & \multicolumn{1}{c|}{$\begin{array}{c}\text { Alumnos } \\
\text { Total }\end{array}$} \\
\hline Instituto Tecnológico de Administración de Empresas, INTAE & 1,087 \\
\hline Instituto Técnico Honduras & 3,099 \\
\hline Instituto Técnico Luis Bográn & 2,688 \\
\hline Instituto Técnico Cultura Popular & 720 \\
\hline Instituto Abelardo Fortín & 2,188 \\
\hline Instituto Técnico Saúl Zelaya Jiménez & 3,128 \\
\hline Instituto Técnico en Electricidad, Electrónica y Computación & 404 \\
\hline
\end{tabular}


La denominación "técnico" los distingue del resto de los institutos básicamente por las modalidades que imparten; esto igualmente se conoce como educación vocacional. Tradicionalmente los institutos de educación media imparten el Bachillerato en Ciencias y Letras, en tanto que los institutos de educación vocacional imparten modalidades que requieren de una infraestructura especial y diferente del resto de los colegios, para poder impartir sus clases.

Es importante aclarar que a la fecha el Programa de Apoyo a la Enseñanza Media en Honduras (PRAEMHO) financiado por la Unión Europea, realizó una serie de equipamientos a estos centros, entre los que se encontraba el Instituto Saúl Zelaya Jiménez. Asimismo, en 2006 se realizó un programa de equipamiento de institutos técnicos e industriales entre los que figuraron el Instituto Técnico Honduras, Instituto Técnico Luis Bográn, Instituto Abelardo Fortín, Instituto Saúl Zelaya Jiménez. Pero entre 1990 y 2003 estos institutos carecían prácticamente de una infraestructura adecuada.

El total de los centros en estudio cuentan con al menos una línea telefónica. Sólo un instituto reporta contar con su propia dirección de correo electrónico. El 70 por ciento de lo institutos cuentan con un tipo de acceso Internet, siendo el mayormente mencionado la conexión por cable. Asimismo manifestaron que tienen este acceso disponible para alumnos en los laboratorios y para uso administrativo. El 86 por cierto de los colegios afirmaron contar con su respectivo laboratorio de informática y con una sala audiovisual equipada con su respectivo proyector, las cuales están a disposición de las diferentes asignaturas que las necesiten.

Todos los institutos reconocen contar con una organización de padres de familia, la cual sesiona para conocer y dictaminar sobre asuntos relacionados con el alumnado, en la mayoría de los casos cada dos meses. En cuanto a si existe relación con el patronato de la comunidad o barrio donde están ubicados los centros, la mayor parte ha mencionado que han tenido algún tipo de contacto pero no permanente. Sin embargo, todos realizan actividades comunitarias, como siembra de árboles, recolección de basura en áreas verdes, y otros trabajos puntuales de ornamento comunal.

En la entrevista se hizo la pregunta "Según su criterio ¿Cuál es la finalidad de la educación técnica que se imparte en este instituto? a la cual todos los directores entrevistados coincidieron en que es la formación del alumno para que se inserte exitosamente en el mercado laboral y para prepararlo para ingresar a la universidad. Según los entrevistados, la idea de que la educación técnica forma estudiantes con bases académicas sólidas, ha provocado que los padres prefieran que sus hijos 
cursen este tipo de educación para ingresar a la universidad en carreras afines, pero que según estudios que se han hecho no siempre el estudiante sigue esta iniciativa y se inclina por otras carreras no relacionadas con sus estudios secundarios. De igual manera se les consultó sobre los objetivos ocupacionales de la educación técnica, a la cual coinciden todos en que, a) se les forma para el ejercicio de una profesión especifica, b) se les forma para que puedan emprender su propia empresa. Particularmente en el INTAE se le brinda importancia fundamental a la creación de pequeñas empresas a través de realización de prácticas profesionales en el proceso de creación y gestión de microempresa.

La principal herramienta para la inserción laboral que se utiliza en los colegios técnicos es la Práctica Profesional Supervisada. Todos los Directores coincidieron en que ésta es una herramienta que ayuda a los alumnos a la puesta en práctica de todo el conocimiento teórico recibido en las aulas. Al menos 6 de los 7 institutos consultados han recibido solicitudes de contratación dirigidas a los egresados, de parte de empresas. Estos contactos han sido de carácter permanente a través de los años, según los entrevistados, y se respaldan por la credibilidad en la educación que imparten dichos centros.

Lo interesante es que a pesar de no contar con una política de inserción laboral como tal, los institutos se las han arreglado para tener una buena efectividad de inserción laboral de sus egresados, a través según ellos, de la calidad de la educación. Ésta ha sido prácticamente la carta de presentación que han utilizado por años para lograr este objetivo.

\section{CONCLUSIONES}

1) En el Distrito Central durante el período 1990-2006, las enormes cifras de desempleados y subempleados jóvenes provocan condiciones de precariedad, inestabilidad y flexibilización de los que están empleados, y este es el efecto del modelo de acumulación capitalista existente en el país.

2) En el Distrito Central, la mayor proporción de jóvenes entre 15 y 24 años empleados, ostentan niveles de educación media sobre todo en las modalidades de Perito Mercantil, Bachillerato en Computación, pero al mismo tiempo se emplean en actividades económicas comerciales y de servicios, en las cuales imperan elevadas condiciones de informalidad, precariedad e inestabilidad laboral.

3) Las modalidades técnicas de orientación industrial son las de menor matricula coincidiendo esto con la tendencia a la tercerización tanto de la economía como de orientación profesional. 
4) La mitad de los docentes no poseen titulo profesional de acuerdo a los requerimientos establecidos para desempeñarse en educación media; no siempre aplican los conocimientos en la forma adecuada debido en parte a la falta de profesionalización continua, secciones supernumerarias, condiciones de baja infraestructura de los institutos y las constantes interrupciones del periodo lectivo.

5) La situación de los colegios técnicos en relación con la infraestructura es significativamente inadecuada; las estructuras curriculares en proceso de transformación, la falta de programas de acompañamiento e inserción laboral y debilidad institucional para el establecimiento de alianzas estratégicas con el sector productivo, condicionan a una formación técnico profesional desorientada, desarticulada y sin pertinencia.

6) El estudio permite probar la hipótesis central, en tanto revela que la baja calificación técnica de los estudiantes de secundaria les está condicionando a puestos de menor nivel y con bajos salarios, en tanto que los empleados no gozan de la estabilidad y condiciones mínimas de un empleo digno y productivo.

\section{BIBLIOGRAFÍA}

Banco Central de Honduras. Honduras en Cifras 1990-2006. Tegucigalpa: 2005.

Batisda, Antonio, (editor). La inserción socio-laboral, reflexiones sobre la práctica. Madrid: Popular; 2001.

Charmes, Jacques. El empleo en el sector informal. Su integración a las estructuras económicas. Buenos Aires: Hvmanista; 1992.

Foro Nacional de Convergencia. Currículo Nacional Básico Honduras 2004. [Documento electrónico] Tegucigalpa: 2004. Disponible en:

http://www.se.gob.hn/content_htm/pdfs/cnb/cnb.pdf

Fundación Santillana. Aprender para el futuro. La Educación Secundaria, pivote del sistema educativo. Madrid: Santillana; 1998.

Giménez, Gregorio. La dotación de capital humano de América Latina y El Caribe. Revista de la CEPAL. 2005; (86): 103-122. [Versión electrónica]. Disponible en: http://www.cepal.org/publicaciones/xml/4/22214/G2282eGimenez.pdf

Gómez, Víctor. Educación para el trabajo: Un estadio sobre la educación técnica industrial. Santafé de Bogotá: Cooperativa Editorial Magisterio. 1998.

Hernández, Rusbell. Estudio sobre la educación para la población rural en Honduras. [Versión electrónica]. Tegucigalpa: 2003. Disponible en:

http://www.red-ler.org/estudio_education:poblacion_rural_honduras.pdf Honduras. Secretaría de Educación. Memorias (1991-2006). Tegucigalpa.

Honduras. Secretaría de Educación. Educación y desarrollo. Estudio sectorial Plan Decenal. Tegucigalpa: 1997. 
Honduras. Secretaría de Educación. Tendencias actuales en la formación técnicoprofesional en América Latina y Hondura. [Versión electrónica]. 2006.

Disponible en:

http://www.oei.es/pdfs/tendencias_ftp_honduras.pdf

Honduras. Secretaría de Educación Pública. Plan Nacional de Desarrollo Educativo 1994-1997. Tegucigalpa: 1994.

Marx, Carlos. El Capital Critica de la Economía Política. México: Fondo de Cultura Económica. 1946. Vol. 1.

Organización Internacional del Trabajo. Tendencias mundiales del empleo juvenil. Ginebra. 2004. [Versión Electrónica]. Disponible en:

http://www.ilo.org/public/spanish/region/ampro/cinterfor/temas/youth/ doc/cint/tmej.htm

Organización Internacional del Trabajo. Informe de la OIT. [Versión electrónica]. 2006. Disponible en:

http://www.oit.org.pe/index.php?option=com_content\&view=article\&id= 292\%3Ael-desempleo-juvenil-aumenta-en-todo-el-mundo\&catid= $117 \% 3$ Aultimas-noticias\&Itemid=1305\&limitstart=1

PNUD. Informe sobre Desarrollo Humano 2006. Barcelona: Aedos. 2006.

PNUD. Informe sobre Desarrollo Humano Honduras 2006. Tegucigalpa: 2006.

PNUD. Objetivos de Desarrollo del Milenio. Segundo Informe de país 2007. Tegucigalpa: 2007.

PRAEMHO. Propuesta Estructura de nuevo Diseño Curricular 2006. [Versión electrónica]. Disponible en: http://www.se.gob.hn

Salgado, R., Soleno, R. Reformas Educativas en Honduras. 1ra. ed. Tegucigalpa: Fondo Editorial. 2002.

UMCE. Informe sobre factores asociados al rendimiento académico Honduras 2002. Tegucigalpa: 2002.

Visauta V, Bienvenido. Análisis Estadístico con SPSS 14. 3ra. ed. Madrid: McGraw-Hill. 2007. 